\title{
ORIGINAL RESEARCH \\ Mechanical Thrombectomy in Acute Ischemic Stroke: Catch Device
}

\section{Mourand \\ H. Brunel \\ V. Costalat \\ C. Riquelme \\ K. Lobotesis \\ D. Milhaud \\ C. Héroum \\ C. Arquizan \\ M. Moynier}

A. Bonafé
BACKGROUND AND PURPOSE: Mechanical thrombectomy presents today a promising alternative to traditional stroke therapies. Our aim with this study was to evaluate the safety and efficacy of the Catch mechanical thrombectomy device in the treatment of acute stroke and report the angiographic results and clinical outcomes.

MATERIALS AND METHODS: We performed an analysis of 40 consecutive patients with ischemic stroke treated with the Catch device at our academic center. Forty patients were treated with the device: 25 with anterior circulation stroke and 15 with posterior circulation stroke. Thirty seven (92.5\%) patients received an additional treatment to aid revascularization, including 36 patients treated with rtPA (mean dose of $35 \mathrm{mg}$ ).

RESULTS: The mean age was 63.5 years (range, 31-82 years; $55 \%$ men). The median NIHSS score at presentation was 18 (range, 3-38). The mean symptom-to-procedure start time was 440 minutes (range, 150-2637 minutes). Recanalization (TIMI 2-3) was achieved in 26/40 (65\%). Symptomatic hemorrhage occurred in $18 \%$. Procedural complications occurred in 6 patients without clinical consequences: 4 clot fragmentations and 2 vasospasms. Data were missing for 4 patients at 90 days. Ninety-day mortality was $41 \%$; good 90-day functional outcome (mRS, $\leq 2$ ) was achieved by $39 \%$ (14/36). Good neurologic outcomes at 90 days were more frequent (56.5\% versus $7.7 \%$ ), and mortality rates were lower (30\% versus $61.5 \%$ ) with successful compared with unsuccessful recanalization.

CONCLUSIONS: In our retrospective case series, the Catch device appears effective in achieving recanalization and improving 90-day outcome in patients with acute ischemic stroke.
$E$ ndovascular mechanical thrombectomy today offers the promise of an alternative and synergistic method of treatment to thrombolysis in acute ischemic stroke. It has several theoretic advantages, such as the ability to deliver site-specific therapy, tailored thrombolytic dosage and delivery, extended treatment time windows, and higher recanalization rates. It also can be used as a rescue therapy in patients who have not responded to IV rtPA or as a combined therapy with other endovascular techniques. Studies report a benefit from mechanical thrombectomy compared with thrombolysis ${ }^{1}$ or from combining IV rtPA pretreatment with mechanical thrombectomy. ${ }^{2-5}$ In 2004, the Merci retriever (Concentric Medical, Mountain View, California) was the first stroke device to be approved by the US Food and Drug Administration and thus started the era of mechanical thrombectomy. An increasing number of different mechanical thrombectomy devices are currently being introduced. The Catch thrombectomy device (Balt, Montmorency, France) is a self-expanding

Received September 29, 2010; accepted after revision November 20.

From the Departments of Neurology (I.M., D.M., C.H., C.A.) and Neuroradiology (H.B., V.C., K.L., C.R., M.M., A.B.), University Hospital Center of Montpellier, Gui de Chauliac Hospital, Montpellier Cedex, France.

Please address correspondence to Isabelle Mourand, MD, Service de Neurologie, Hôpital Gui de Chauliac, 80 Ave Augustin Fliche, 34295 Montpellier Cedex 5, France; e-mail: i-mourand@chu-montpellier.fr

http://dx.doi.org/10.3174/ajnr.A2563 nitinol basket that has also been used for thrombectomy with promising results. ${ }^{6}$

In an attempt to offer an additional/alternative therapeutic option for patients with acute ischemic stroke and a major vessel occlusion presenting outside the IV therapeutic time windows or with contraindications to IV fibrinolysis, the Catch thrombectomy device was used in our academic center. The purpose of this study was to retrospectively evaluate the efficacy and safety of the Catch thromboembolectomy system in a consecutive series of 40 patients with ischemic stroke treated in our center. We present the immediate angiographic and technical results with regard to the use of the device as well as the clinical outcome at 3 months posttreatment.

\section{Materials and Methods}

We retrospectively reviewed 40 consecutive patients who were treated for acute ischemic stroke and underwent mechanical thrombectomy between September 2005 and July 2008 (34 months).

Patients were considered for mechanical thrombectomy for the following reasons: 1) They had experienced an acute ischemic stroke with a significant NIHSS score (ie, $\geq 7$ for anterior circulation strokes [ICA, and MCA occlusions] and no NIHSS limit for posterior circulation occlusions). 2) They presented after the 3-hour time window for IV thrombolysis but before the 6-hour treatment cutoff in anterior circulation strokes and before 24 hours in vertebrobasilar occlusions. 3) They presented within 3 hours of symptom onset but with a systemic contraindication to IV thrombolysis. 4) They presented with a 

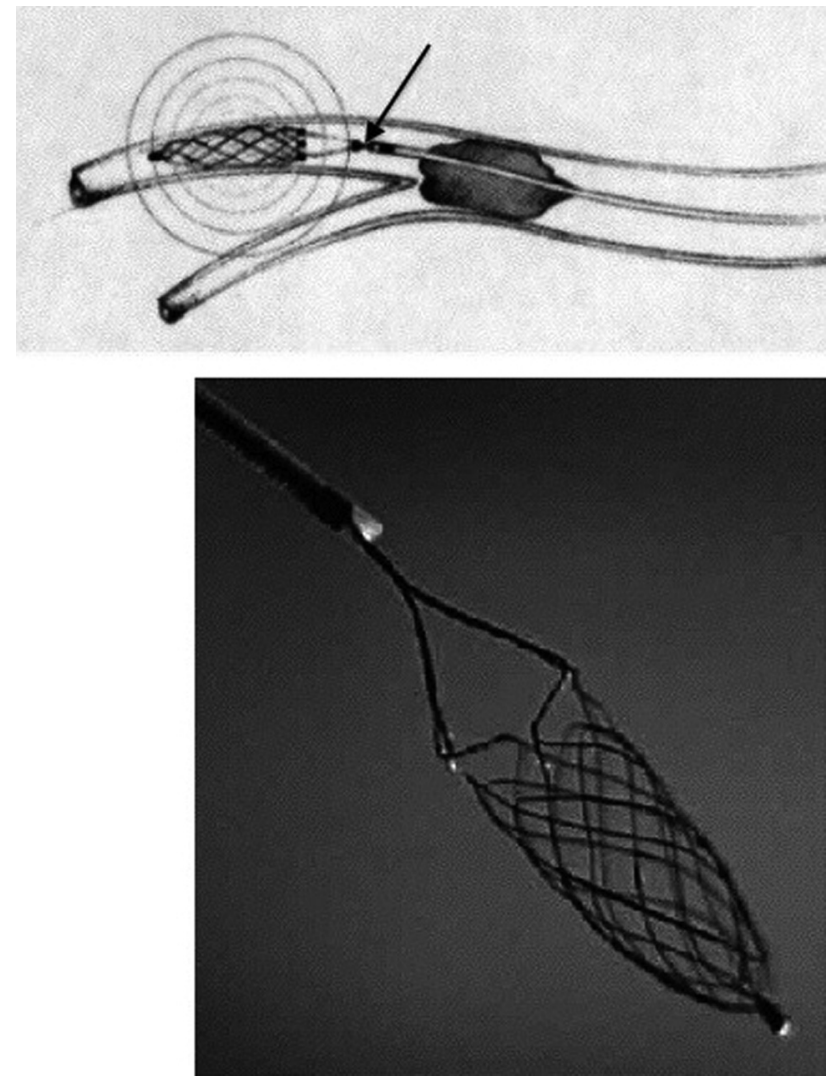

Fig 1. The Catch retriever with the self-expanding basket fixed to a pusher wire (arrow). Courtesy of Balt Extrusion, Montmorency, France.

wake-up stroke but with no changes on the FLAIR sequence for an anterior circulation stroke or within 24 hours for stroke in the posterior circulation. 5) They had MR imaging demonstrating an ischemic lesion and thrombus in a main cerebral artery $(>1.5 \mathrm{~mm})$. Patients were excluded for the following reasons: 1) They were pregnant. 2) They presented with a seizure at stroke onset. 3) They were younger than 18 years of age. 4) They had $<50000$ platelets. 5) They had severe hypertension (defined as systolic blood pressure $>185 \mathrm{~mm} \mathrm{Hg}$ or diastolic blood pressure $>110 \mathrm{~mm} \mathrm{Hg}$ ). 6) They had cerebral imaging revealing intracranial hemorrhage, tumor, subacute infarct, significant mass effect with midline shift, or a large ischemic lesion of more than half the MCA territory.

Patients who met all clinical and radiologic criteria listed above and for whom informed consent was obtained, underwent diagnostic cerebral angiography. This was performed through a femoral artery approach with the patient under general anesthesia. Patients were selected if the angiogram showed an occlusion of the ICA (proximal and terminal segments), MCA (including M1 or proximal M2 segments), BA, or VA. Endovascular treatment was performed under systemic heparinization (2500-UI bolus).

All patients were treated with the Catch thromboembolectomy system. The Catch device in the European Union is Communaute Européenne-certified. Its use in our stroke unit for thrombectomy was approved by the members of our multidisciplinary stroke team; given that this was a retrospective study, no ethics approval was necessary. This system consists of a self-expanding basket-like device with a maximum diameter of $4 \mathrm{~mm}$, which is fixed to a pusher wire (Fig 1). Using standard cerebral catheterization techniques, we navigated a microwire and a Vasco21 microcatheter (Balt) into the oc- cluded vessel and passed them beyond the thrombus. A selective angiogram was obtained distal to the thrombus. The Catch device was then advanced through the microcatheter and deployed distal to the thrombus. Both the basket and the microcatheter were then simultaneously pulled back, engaging the thrombus and retracting it into the guide catheter. Although the device was used without proximal balloon occlusion, aspiration was performed during the Catch retrieval. The number of attempts to retrieve the thrombus was limited to 5 per occluded vessel. The use of treatments or devices additional to the Catch device was left to the discretion of the interventional neuroradiologist. These included IV or IA thrombolysis, thromboaspiration, angioplasty, and stent placement in patients with a persisting occlusion.

All patients were admitted to a neurointensive care or stroke unit posttreatment. Concomitant antithrombotic therapy was not used. No patients were treated with GIIb/IIIa inhibitors. All antiplatelet therapies were started 24 hours after the procedure and after completion and review of the 24-hour cerebral CT for evidence of hemorrhage.

Outcome measures included arterial recanalization according to the TIMI scale, ${ }^{7}$ presence of symptomatic and asymptomatic hemorrhage, 90-day mRS, in-hospital mortality, and 90-day mortality. The goal of thrombectomy was to achieve successful recanalization, defined as TIMI grade 2 or 3. Symptomatic intracerebral hemorrhage was defined as a homogeneous area of hemorrhage on CT, with neurologic deterioration defined as an increase in NIHSS of $\geq 4$ points. Intracerebral hemorrhages were also classified radiologically according to the Berger scale. ${ }^{8}$ Favorable outcome was defined as a 90 -day mRS score of $0-2$.

\section{Results}

During the study period, 40 consecutive patients with acute ischemic stroke met the inclusion/exclusion criteria listed above and underwent urgent cerebral angiography and thrombectomy with the Catch system. The Catch was the unique device used for thrombectomy in our center during this period.

The mean age of the cohort was 63.5 years (range, 31-82 years), with $55 \%$ men and $45 \%$ women ( 22 men, 18 women). Target clot sites were the anterior circulation in $25(62.5 \%)$ and the posterior circulation in $15(37.5 \%)$ patients. Occluded arteries included the MCA in 16 patients (40\%), the ICA in 9 patients $(22.5 \%)$, the BA in 14 patients $(35 \%)$, and the VA in 1 patient $(2.5 \%)$.

The overall pretreatment NIHSS score was 18 (range, 3-38) with a score of 17 (range, 9-23) for anterior circulation and 21 (range, 3-38) for posterior circulation occlusions. A score of 38 was assigned when NIHSS was performed on sedated patients.

For the 40 patients with stroke, the mean time from symptom onset to procedure start was 440 minutes ( 7 hours and 20 minutes). Three patients presented within 3 hours of symptom onset but had a systemic contraindication for IV rtPA.

Overall, successful recanalization (TIMI 2-3) occurred in $65 \%(26 / 40)$. More specifically, TIMI 3 was achieved in $32.5 \%$ (13/40), and TIMI 2, in 32.5\% (13/40). Recanalization by target vessel location was MCA $69 \%$ (11/16), ICA 44\% (4/9), BA $71 \%(10 / 14)$, and VA $100 \%(1 / 1)$, with a poor rate for the ICA but a good rate for the posterior circulation.

All patients included in this study were treated with the 

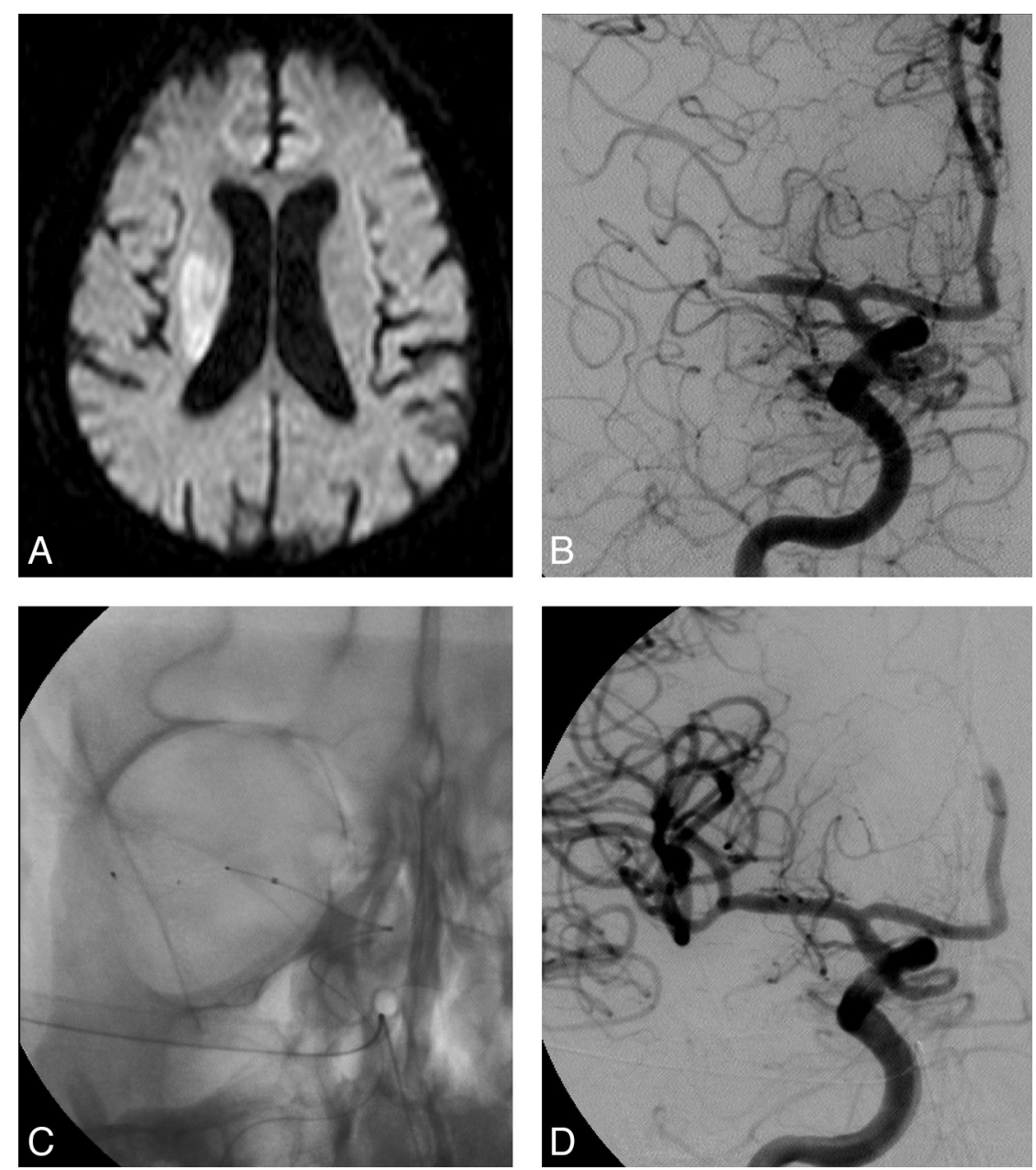

Fig 2. A 75-year-old man with left-sided weakness (NIHSS score, 18) who presented to our stroke unit and underwent MR imaging at 1.5 hours after symptom onset. $A$, Diffusion-weighted imaging sequences demonstrate an acute right MCA infarct involving mainly the right basal ganglia. $B$, Initial angiogram obtained 3 hours after the onset of his symptoms confirms a right MCA occlusion. $C$ and $D$, After 4 passes with the Catch device, complete recanalization is achieved without additional treatment needed. At 90-day follow-up, he had an NIHSS score of 3 with an mRS score of 2 .

Catch thromboembolectomy system. The average number of passes of the device was 2 (range, 1-5). Thirty-seven patients $(92.5 \%)$ received an additional treatment to aid revascularization: IV or IA rtPA in $90 \%$ (36/40), thromboaspiration by the catheter in 10\% (4/40), angioplasty in 30\% (12/40), and stent placement for residual stenosis in 10\% (4/40). Of the 3 patients treated with the Catch device alone, 2 had contraindications to rtPA and 1 demonstrated complete recanalization at the end of the mechanical embolectomy (Fig 2). Among the 36 patients who received rtPA, 5 received IV alone (14\%); 26, IA (72\%); and 5, both IV and IA (14\%). Among the 10 patients who received IV rtPA, 4 received it before and 6, after mechanical thrombectomy. Thirty-one received IA rtPA in an attempt to treat residual thrombus following thrombectomy. The mean dose of rtPA was $35 \mathrm{mg}$ (range, $10-50 \mathrm{mg}$ ).

Procedural complications occurred in $6(15 \%)$ patients without clinical consequence, including 4 clot fragmentations with distal embolism and 2 vasospasms treated by an IA infusion of nimodipine. There were no cases of procedure-related ischemia in a new vascular territory and no vessel dissections or perforations.

All patients except 1 underwent CT at 24 hours to assess hemorrhage. Symptomatic hemorrhage occurred in 7 of the
39 patients (18\%). One of the 7 patients had a subdural hematoma with mass effect, 5 patients had intraparenchymal hemorrhage and 1 had an SAH and an intraparenchymal hemorrhage. Of these 6 patients with intraparenchymal hemorrhage, 4 had large hematomas with mass effect (parenchymal hematoma type 2) contributing to the death of the patients. Overall 6 of the 7 patients with symptomatic hemorrhage died. Asymptomatic hemorrhage occurred in 12 patients $(31 \%)$ : One of these hemorrhages was an $\mathrm{SAH}$, and none were parenchymal hematoma type 2 .

In our cohort, 4 patients were lost for follow-up after the in-hospital period. Overall, good 90-day functional outcome (mRS, $0-2$ ) was achieved in 14 patients $(14 / 36,39 \%)$. This outcome occurred in 13 recanalized patients and 1 nonrecanalized patient (56.5\% versus $7.7 \%$ ). Good outcome occurred in $41 \%$ of patients who experienced ischemia in the anterior circulation (9/22), and in $36 \%$ in the vertebrobasilar territory (5/14). The overall in-hospital mortality rate was $37.5 \%$, with a rate of $32 \%$ when the stroke involved the anterior circulation $(8 / 25)$ and $47 \%$ for vertebrobasilar occlusion (7/15). The $90-$ day mortality rate was $41 \%(15 / 36)$. Among the 11 deaths (73\%) due to neurologic causes, 8 occurred within 6 days; the causes included brain edema, cerebral hemorrhage, and stroke 


\begin{tabular}{lc}
\hline NIHSS, recanalization, and outcome in our patients & \\
\hline Overall Outcome & 18 \\
\hline Baseline NIHSS score (median) & $65 \%$ \\
Revascularization & \\
Favorable outcome (90-day) & $39 \%$ \\
$\quad$ mRS score $\leq 2$ & $32 \%$ \\
NIHSS score improved from baseline by $\geq 10$ points & $41 \%$ \\
Mortality (90-day) & $18 \%$ \\
Symptomatic hemorrhage &
\end{tabular}

recurrence. Favorable neurologic outcome at 90 days was associated more with successful vessel recanalization compared with unsuccessful recanalization ( $56.5 \%$ versus $7.7 \%)$, as were lower mortality rates (30\% versus $61.5 \%$ ). Overall outcome is presented in the Table.

\section{Discussion}

In this study, we report an overall successful recanalization rate (TIMI 2-3) in 65\% of patients treated with Catch and adjunctive therapy. The Catch device was used alone in 3 patients, with successful recanalization achieved in 2 of them. These results are comparable with available data from the PROACT II ${ }^{9}$ and Multi MERCI trials, ${ }^{10}$ which demonstrated $66 \%$ and $68 \%$ recanalization rates of TIMI 2 and 3, respectively. Moreover, the recanalization rates observed in a recent meta-analysis performed by Rha and Saver ${ }^{11}$ included 53 studies and 2066 patients. The authors reported spontaneous recanalization $(24.1 \%)$, IV thrombolysis (46.2\%), IA thrombolysis $(63.2 \%)$, combined IV/IA thrombolysis (67.5\%), and mechanical treatment (83.6\%). The predominant mechanical therapy used in the series was intracranial angioplasty.

A number of devices are currently commercially available for mechanical thromboembolectomy. In a recent study comparing the performance of 5 different embolectomy systems, by using an in vitro pulsatile flow model, the Merci, Catch, and Phenox (Phenox, Bochum, Germany) retrievers were equally able to mobilize and remove most thrombi, whereas the InTime and Attractor (Boston Scientific, Natick, Massachusetts) devices achieved only a partial thrombus removal at best and with considerable difficulty during initial thrombus penetration and placement of the device. ${ }^{12}$

To improve thrombus retrieval, we used the Catch in association with other endovascular treatment or IV rtPA (37/40 patients, 92\%). The most frequent additional treatment was IV and/or IA rtPA (36/40, 90\%), with a mean dose of $35 \mathrm{mg}$. Other therapies included thromboaspiration, angioplasty, and stent placement. In our cohort, 35\% of patients received at least 3 treatments; mostly, these were patients with carotid artery (7/9 of the total treated carotid arteries, $77 \%)$ and vertebrobasilar artery occlusions $(6 / 15,40 \%)$, and 1 patient with MCA occlusion $(1 / 16,6 \%)$. In the Multi MERCI trial, ${ }^{10} 48$ patients $(29.3 \%)$ received IV rtPA before intervention, and 57 patients, intraprocedural IA lytics. Treatment with the retriever alone resulted in successful (TIMI 2-3) recanalization in $55 \%$ of treatable vessels and in $68 \%$ after adjunctive therapy (IA rtPA, mechanical). As in other studies, ${ }^{13,14}$ in our cohort, several attempts were necessary to achieve recanalization, with an average number of 2 passes (range, $1-5$ ). As with all devices, time is required for preparation of the device before re-use.
This was estimated to be on the order of 5-10 minutes for the Catch device.

Our overall 90-day good functional and mortality rates were $39 \%$ and $41 \%$, respectively. In the PROACT II, ${ }^{9}$ MERCI, ${ }^{3}$ and Multi MERCI ${ }^{10}$ trials, the good clinical outcome rates were $40 \%, 27.7 \%$, and $36 \%$, respectively. Among our 23 patients who were revascularized, 13 made a significant recovery (defined as 90-day mRS score of 0-2). In contrast, among the 13 patients not revascularized, only 1 made a significant recovery. As in the $\mathrm{MERCI}^{3}$ and Multi MERCI ${ }^{10}$ trials, we report higher good neurologic outcomes at 90 days $(56.5 \%$ versus $7.7 \%$ ) and lower mortality rates (30\% versus $61.5 \%$ ) with successful compared with unsuccessful recanalization. This strong correlation between vessel recanalization and favorable outcome was further highlighted in the previous meta-analysis, ${ }^{11}$ which concluded that good functional outcomes at 3 months were more frequent in recanalized versus nonrecanalized patients (odds ratio, 4.43), 3-month mortality was reduced in recanalized patients (odds ratio, 0.24), and rates of symptomatic hemorrhagic transformation did not differ between the 2 groups.

Patients treated in the NINDS, ${ }^{15}$ PROACT II trial, ${ }^{9}$ MERCI, $^{3}$ and Multi MERCI ${ }^{10}$ trials had mortality rates of $12.5 \%, 25 \%, 43.5 \%$, and $34 \%$ respectively. In our cohort, the mortality rate of $41 \%$ is comparable with the Merci rate and could be explained by the longer window for treatment and the inclusion of most patients with posterior circulation strokes. Our patients were included up to 6 hours if they had a stroke in the anterior circulation and up to 12 hours if they had a stroke in the posterior circulation. A stroke involving vertebrobasilar arteries occurred in $37.5 \%$ of our patients in comparison with $10 \%$ and $8 \%$ in the $\mathrm{MERCI}^{3}$ and Multi MERCI ${ }^{10}$ trials, respectively. The mortality rate observed in our study varied considering the target clot sites: anterior circulation, $32 \%$; posterior circulation, $47 \%$. In a pooled analysis of the patients with BA occlusion enrolled in the $\mathrm{MERCI}^{3}$ and Multi $\mathrm{MERCI}^{10}$ trials treated with the Merci retriever within 8 hours after symptom onset, recanalization occurred in 21 of 27 (78\%) patients, mortality was $44 \%$, and favorable outcomes ( $\mathrm{mRS}$ score, $0-3$ ) were seen in $41 \% .{ }^{16}$ In this report, the failure to recanalize and the presence of coma on presentation were associated with higher mortality rates, whereas thrombus localization in the distal third of the BA, compared with the proximal and/or middle portions of the BA, was associated with a lower mortality rate. In our population of vertebrobasilar artery occlusions, we report successful recanalization (TIMI 2-3) in 73\%, mortality in $47 \%$, and favorable outcome (mRS score, $0-3$ ) in 50\%. Thus, no relation between mortality, revascularization, or coma on presentation was found.

Our rate of symptomatic hemorrhage $(18 \%, 7 / 39)$ was higher compared with previously reported rates for the National Institute of Neurological Disorders and Stroke (6.4\%), ${ }^{15}$ PROACT II (10\%), ${ }^{9}$ MERCI $(7.8 \%),{ }^{3}$ and Multi MERCI trials (9.8\%). ${ }^{10}$ The outcome for patients that developed hemorrhagic complications was unfavorable with an mRS $>2$ of $94 \%$ and death of $70 \%$ at 90 days. Six patients presented with symptomatic hemorrhages and 4 (10\%) developed parenchymal hematomas type 2. Two SAHs occurred in our cohort, both associated with intraparenchymal hemorrhage. In neither case was vascular perforation or contrast ex- 
travasation observed during the procedure. We hypothesize a possible revascularization cause for our SAHs, though an intimal damage etiology cannot be excluded. SAH was also a recognized complication of the MERCI trial but was considered a result of vascular perforation. ${ }^{3}$

Among the common complications of the retrieval, we observed 4 thrombus fragmentations with distal embolization without procedure-related ischemia in a new vascular territory, even if we did not use balloon protection or aspiration. All devices produced micro- and macrofragments during thrombus penetration and retrieval. It has been demonstrated, in an animal model, ${ }^{13,14}$ that the Catch device increased the risk of vessel wall irritation significantly and the number of distal embolic events compared with Merci (40\% versus 10\%) or Vasco35 retrieval (Balt). In the study by Liebig et al, ${ }^{12}$ the Phenox retriever had a superior performance in terms of preventing distal embolization because it was able to capture most of these clot fragments. Embolization can be considerably reduced by using proximal balloon occlusion and aspiration during retrieval.

This study is a consecutive retrospective study with inherent limitations by design. Therefore, our results should be considered as preliminary. In our center, the techniques and timing of mechanical clot disruption are still evolving, given our acquired experience and considering the results of the European Cooperative Acute Stroke Study III trial. ${ }^{17}$

\section{Conclusions}

In our early experience with the Catch retriever, it appears an efficient recanalization device in patients with acute stroke who are either ineligible or have failed to recanalize following rtPA therapy. Using an additional treatment, which was, in most cases, a low mean dose of rtPA, we achieved a high rate of successful recanalization, which is comparable with available data, even in cases of BA or ICA occlusion. Here, good clinical outcome was comparable with available data despite high rates of mortality and symptomatic hemorrhage. Techniques and devices continue to evolve, and randomized clinical trials are needed to demonstrate that endovascular mechanical embolectomy improves patient outcome in acute ischemic stroke.

\section{References}

1. Noser EA, Shaltoni HM, Hall CE, et al. Aggressive mechanical clot disruption: a safe adjunct to thrombolytic therapy in acute stroke? Stroke 2005;36:292-96

2. Gobin YP, Starkman S, Duckwiler GR, et al. MERCI: a phase 1 study of Mechanical Embolus Removal in Cerebral Ischemia. Stroke 2004;35:2848-54

3. Smith WS, Sung G, Starkman S, et al. Safety and efficacy of mechanical embolectomy in acute ischemic stroke: results of the MERCI trial. Stroke 2005;36:1432-38

4. Smith WS, for the Multi MERCI investigators. Safety of mechanical thrombectomy and intravenous tissue plasminogen activator in acute ischemic stroke: results of the Multi Mechanical Embolus Removal in Cerebral Ischemia (MERCI) trial, part 1. AJNR Am J Neuroradiol 2006;27:1177-82

5. Kim D, Jahan R, Starkman S, et al. Endovascular mechanical clot retrieval in a broad ischemic stroke cohort. AJNR Am J Neuroradiol 2006;27:2048-52

6. Chapot R. First experience with the Catch, a new device for cerebral thrombectomy. Intervent Neuroradiol 2005;11(suppl 2):58

7. Williams DO, Borer J, Braunwald E, et al. Intravenous recombinant tissue-type plasminogen activator in patients with acute myocardial infarction: a report from the NHLBI Thrombolysis in Myocardial Infarction trial. Circulation 1986;73:338-46

8. Berger C, Fiorelli M, Steiner T, et al. Hemorrhagic transformation of ischemic brain tissue: asymptomatic or symptomatic? Stroke 2001;32:1330-35

9. Furlan A, Higashida R, Wechsler L, et al. Intra-arterial prourokinase for acute ischemic stroke: the PROACT II study-a randomized controlled trial. Prolyse in Acute Cerebral Thromboembolism. JAMA 1999;282:2003-11

10. Smith WS, Sung G, Saver L, et al. Mechanical thrombectomy for acute ischemic stroke: final results of the Multi MERCI trial. Stroke 2008;39:1205-12. Epub 2008 Feb 28

11. Rha JH, Saver JL. The impact of recanalization on ischemic stroke outcome: a meta-analysis. Stroke 2007;38:967-73

12. Liebig $T$, Reinartz J, Hannes $R$, et al. Comparative in vitro study of five mechanical embolectomy systems: effectiveness of clot removal and risk of distal embolization. Neuroradiology 2008;50:43-52

13. Gralla J, Schroth G, Remonda L, et al. Mechanical thrombectomy for acute ischemic stroke: thrombus-device interaction, efficiency, and complications in vivo. Stroke 2006;37:3019-24

14. Brekenfeld C, Schroth G, El-Koussy M, et al. Mechanical thromboembolectomy for acute ischemic stroke: comparison of the Catch thrombectomy device and the Merci Retriever in vivo. Stroke 2008;39:1213-19. Epub 2008 Feb 28

15. Tissue plasminogen activator for acute ischemic stroke: the National Institute of Neurological Disorders and Stroke rtPA Stroke Study Group. N Engl J Med 1995;333:1581-87

16. Lutsep HL, Rymer MM, Nesbit GM. Vertebrobasilar revascularization rates and outcomes in the MERCI and the Multi MERCI trials. J Stroke Cerebrovascular Dis 2008; 17:55-57

17. Hake W, Kaste M, Bluhmki E, et al. Thrombolysis with alteplase 3 to 4.5 hours after acute ischemic stroke. N Engl J Med 2008;359:1317-29 